\title{
Außenwirtschaft in Zeiten der Globalisierung - Möglichkeiten und Grenzen der statistischen Messung
}

\author{
Hans-Peter Glaab · Joachim Wagner • Markus Zwick
}

Online: 6. Oktober 2009

(C) Springer 2009

Ursachen und Auswirkungen der Globalisierung sowie ihre statistische Erfassung werden seit vielen Jahren auf nationaler und internationaler Ebene diskutiert. Die statistische Erfassung der Globalisierung ist aufgrund der immer komplexer werdenden Produktions- und Finanzstrukturen eine große Herausforderung. Im Rahmen der Reihe „Dialog von Wissenschaft und amtlicher Statistik“ veranstaltete der Rat für Sozial- und Wirtschaftsdaten (RatSWD) zusammen mit der Deutschen Bundesbank und dem Statistischen Bundesamt hierzu am 22. und 23. April 2008 in Wiesbaden eine Konferenz mit dem Ziel einer Intensivierung des Dialogs zwischen Datenproduzenten und Datennutzern. In zahlreichen Beiträgen wurden neue Entwicklungen bei der Messung von Aspekten der Internationalisierung der Wirtschaft diskutiert, neu verfügbare Datenbestände vorgestellt und Ergebnisse von empirischen Untersuchungen präsentiert.

Eine Auswahl dieser Tagungsbeiträge wird - in überarbeiteter Form, in die Anregungen aus der Diskussion und Hinweise der Gutachter sowie des Herausgebers der Zeitschrift eingeflossen sind - in diesem und dem nächsten Heft (Band 3, Heft 3, 2009) des Wirtschafts- und Sozialstatistischen Archivs (WiSoStA) veröffentlicht. Dieses Heft beginnt mit einer ausgearbeiteten Version des Grundsatzreferats, mit dem

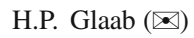

Deutsche Bundesbank, Wilhelm-Epstein-Straße 14, Postfach 1006 02, 60006 Frankfurt am Main, Deutschland

E-Mail: hans-peter.glaab@bundesbank.de

J. Wagner

Leuphana Universität Lüneburg, Postfach 2440, 21314 Lüneburg, Deutschland

E-Mail: wagner@uni-lueneburg.de

M. Zwick

Forschungsdatenzentrum des Statistischen Bundesamtes, Gustav-Stresemann-Ring 11,

65180 Wiesbaden, Deutschland

E-Mail: markus.zwick@destatis.de 
Hermann Remsperger die Konferenz eingeleitet hat. In drei Aufsätzen werden anschliessend Aspekte des Außenhandels mit Waren behandelt. Eine weitere Arbeit untersucht den Einfluss der europäischen Integration auf die Lohnstruktur in der Grenzregion von Bayern und Tschechien.

Hermann Remsperger erörtert in seinem Beitrag ,Globalisierung aus statistischer Sicht" insbesondere die Frage, wie gut vorhandene Statistiken Prozesse der Globalisierung abbilden und welche Herausforderungen sich für die Datenproduzenten im Hinblick auf Anpassungen bestehender und Bereitstellung neuer statistischer Daten ergeben. Er skizziert, welche Daten gegenwärtig verfügbar sind und diskutiert für verschiedene außenwirtschaftliche Statistiken, inwieweit sich durch das Phänomen der Globalisierung die Erhebungsgrundlagen verändert haben und ob - beziehungsweise in welcher Weise - die Aussagefähigkeit der Ergebnisse beeinflusst wird. Schließlich wird an einigen Beispielen dargestellt, welcher neue Datenbedarf durch die Globalisierung entstanden ist. Diese Problematik wird in den weiteren Beiträgen in unterschiedlicher Art und Weise jeweils aus der Sicht von Datenproduzenten und Datennutzern aufgegriffen.

In ihrem Aufsatz „Messung der Globalisierung - Möglichkeiten und Grenzen der Außenhandelsstatistik" widmen sich Klaus Geyer-Schäfer und Albrecht Krockow der Frage, inwieweit sich die Außenhandelsstatistik zur Messung der Globalisierung eignet. Sie zeigen auf, welche Transaktionen die Außenhandelsstatistik erfasst und wo ihre Ergebnisse aufgrund der Globalisierung unzureichend sind bzw. anders interpretiert werden sollten. Sie weisen darauf hin, welche weiteren Datenquellen zur Messung von Globalisierung herangezogen werden können.

Joachim Wagner untersucht in seiner Studie ,Exporte und Firmenerfolg: Welche Firmen profitieren wie vom internationalen Handel?" die Frage, welche Firmen auf welche Weise vom internationalen Handel profitieren. Eine Befragung von exportierenden Firmen zeigt, dass nicht alle in gleicher Weise und in gleichem Maße vom Export profitieren. Starke positive Effekte finden sich bei mehr als der Hälfte der Firmen im Hinblick auf Wachstum, Kapazitätsauslastung und Erträge sowie bei rund 40 Prozent mit Bezug auf den Ausgleich inländischer Konjunkturschwankungen. Die Wahrscheinlichkeit dafür, dass starke positive Effekte zu beobachten sind, steigt mit zunehmendem Anteil des Exportes am Umsatz an.

Die Arbeit „Beschäftigungseffekte von Produkt- und Prozessinnovationen bei Exporteuren und Nicht-Exporteuren" von Sascha O. Becker und Peter H. Egger basiert auf jährlichen Befragungsdaten von Unternehmungen des verarbeitenden Gewerbes, die das Ifo Institut erhebt. 75 Prozent der befragten Unternehmen stehen als Exporteure ganz besonders im globalen Wettbewerb. Becker und Egger gehen der Frage nach, ob Produkt- oder Prozessinnovationen wichtiger für das Beschäftigungswachstum sind. Sie zeigen, dass Produkt- und Prozessinnovationen heterogene Auswirkungen auf das Beschäftigungswachstum aufweisen. Prozessinnovationen erhöhen das Beschäftigungswachstum von Exporteuren und reduzieren jenes von Nicht-Exporteuren. Produktinnovationen erzeugen gegenteilige Wirkungen.

Die Arbeitsmärkte in Grenzregionen zu den mittelosteuropäischen EU-Mitgliedern sind von der wirtschaftlichen Integration Europas besonders betroffen. Michael Moritz untersucht in seiner Studie ,,The Impact of European Integration on Wage Differentials in the Bavarian-Czech Border Region“ den Einfluss der europäischen Integra- 
tion auf die Lohnstruktur in der Grenzregion von Bayern und Tschechien. Auf Basis der Daten der IAB-Beschäftigtenstichprobe und der Beschäftigten-Historik schätzt Moritz die Lohndifferentiale zwischen Arbeitnehmern in der Grenzregion Ostbayern und dem restlichen westdeutschen Bundesgebiet. Nach diesen Schätzungen kam es Anfang der 1990er Jahre für einen Großteil der Arbeitnehmer in Ostbayern zu einem Aufholprozess, während der Lohnabstand seit 1995 wieder wächst.

Das folgende Heft 3 des Jahrgangs 2009 des Wirtschafts- und Sozialstatistischen Archivs (WiSoStA) wird vier Konferenzbeiträge enthalten, die sich mit dem Thema „Direktinvestitionen“ befassen. Sebastian Hügelschäffer, Daniel Kromer und Alexander Lipponer werden neue Entwicklungen bei der deutschen Statistik über Direktinvestitionen vorstellen und einen Einblick in die vielfältige wissenschaftliche Nutzung dieser Daten geben. Peter Schmidt, Bernd Waldmüller, Jörg Feuerhake und Beatrix Stejskal-Passler werden über die künftige Statistik über ausländisch beherrschte Unternehmen in Deutschland und Tochterunternehmen deutscher Investoren im Ausland informieren. Christian Arndt und Anselm Mattes nutzen Daten des IAB-Betriebspanels für eine empirische Untersuchung der Determinanten und Effekte von Direktinvestitionen im Ausland am Beispiel baden-württembergischer Betriebe. Vivien Procher betrachtet Standortentscheidungen von französischen Firmen, die 2004 erstmals als Direktinvestoren ins Ausland gegangen sind.

Fragen im Zusammenhang mit der statistischen Erfassung, den Ursachen und den Folgen der Globalisierung werden noch für lange Zeit - insbesondere in Deutschland, einem wichtigen „Global Player“ auf den internationalen Güter-, Dienstleistungsund Kapitalmärkten - im Zentrum wissenschaftlicher Debatten stehen. Der Dialog zwischen Datenproduzenten und Datennutzern sollte daher auch auf diesem Gebiet intensiv fortgesetzt werden.

Danksagung Unser Dank gilt Dipl.-Soz. Patricia Eilsberger (Destatis) für die Hilfe bei der Vorbereitung und Durchführung der Tagung ,Außenwirtschaft in Zeiten der Globalisierung“, den Gutachtern für die sorgfältige (oft mehrmalige) Lektüre der eingereichten Beiträge zu diesem Heft und Dipl.-Soz. Tim Hochgürtel (Destatis) für seine sehr kompetente Unterstützung des Redaktionsprozesses. Insbesondere danken wir dem Herausgeber Prof. Dr. Hans Wolfgang Brachinger dafür, dass er alle Beiträge ausführlich kommentiert und an vielen Stellen durch zahlreiche Vorschläge deutlich verbessert hat.

Frankfurt, Lüneburg und Wiesbaden, im September 2009

Hans-Peter Glaab, Joachim Wagner, Markus Zwick 